\title{
Significance of Fatigue Damage from Overload Trucks on Bridge Load Rating
}

\author{
Bora Jang ${ }^{1, *}$, and Jamshid Mohammadi ${ }^{2}$ \\ ${ }^{1} \mathrm{PhD}$ Candidate, Department of Civil, Architectural and Environmental Engineering, Illinois Institute of Technology, Chicago, Illinois \\ USA \\ ${ }^{2}$ Professor, Department of Civil, Architectural and Environmental Engineering, Illinois Institute of Technology, Chicago, Illinois USA
}

\begin{abstract}
Overload trucks constitute a major portion of truck load populations on highways. Specific to bridges, the increase in the frequency of overloads may trigger shortening of service life of critical components because of fatigue damage, considering the fact that fatigue damage accelerates at higher loads. This is especially important to older bridges that have been designed for loads lower than the standard for current highway bridge design in the United States. As the number of requests for special permits to use bridges by overload trucks increases, the accumulated damage may substantially reduce the service life of bridges resulting in costly repair and replacements. In this paper, the significance of overloads in causing fatigue damage to bridges is discussed. Samples of truck load data have been compiled and used to determine the contribution of overloads to fatigue damage for steel girder bridges made up of welded cover plates. The paper discusses using the expected fatigue life expended (FLE) as an index to determine the impact of overloads on bridge fatigue damage. The FLE is then determined to be an important factor that can be used to modify bridge rating equations as a way of controlling the number of overload permits for a given bridge.
\end{abstract}

\section{Introduction}

\subsection{Truck load population and overloads}

The intensity and frequency of load occurrences in the truck load population vary to a great extent for a given vehicle class. Load populations mostly exhibit an inconsistent pattern often with two or more distinct peaks. Figure 1 shows the distribution of truck load data, designated as the gross vehicle weight (GVW), for one data sample compiled by weigh-in-motion (WIM) system in Illinois (by the Illinois Department of Transportation (IDOT) from June, 2014 to May, 2015). As seen in Figure 1, the distribution appears with two peaks in the entire truck load population. That is because of the variety of loads in the population. The appearance of two peaks may be due to a combination of loaded and empty trucks in most cases. In addition, the population also contains overload frequencies that may be significant in some cases resulting in multiple peaks in the population. It is noted that the term overload, as used here, refers to truck weights in excess of the 356-kN (80kip) limit. Studies on the effect of truck loads on highway systems have shown that truckload spectra often contain occurrences of overloads [1-3]. In certain cases, overloads are rather frequent; and as such, they may significantly affect the service life of bridges $[1,4]$. Using the weigh-in-motion (WIM) system, truck load data gathered in several stations in Illinois and Michigan indicate that the percentage of overloads can be as high as $10 \%$ of the total truck load population. It is rather large and can be very critical for certain bridges with critical components prone to fatigue damage. A traffic volume for vehicle class 9 in 5-axle truck category is considered as a fatigue loading, since this type of truck appears to make up a significant portion of the entire truck load population.

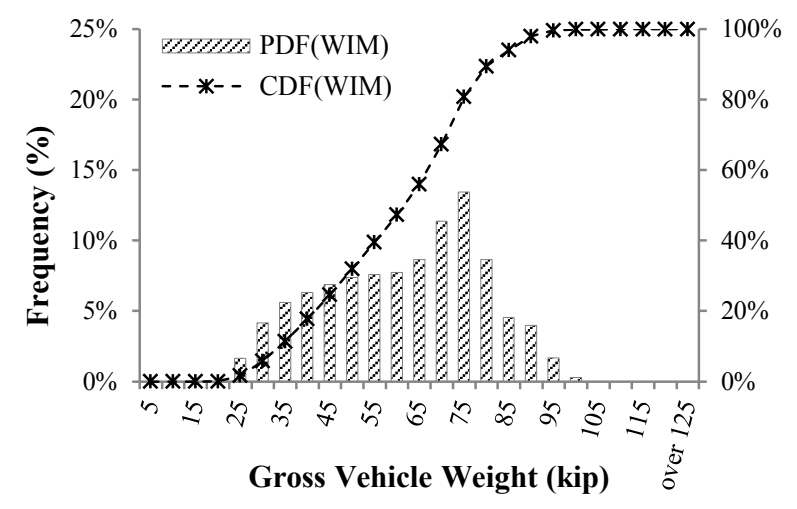

Fig. 1. Frequency distribution of gross vehicle weight for vehicle class 9 in 5-axle truck from WIM data.

\subsection{Fatigue damage potentials}

* Corresponding author: bjang5@hawk.iit.edu 
As it is known, fatigue damage initiates cracks within structural components of bridges. These cracks grow in length with the repeated application of loads leading to a potential failure. Fatigue cracks grow more rapidly as the intensity of truck weights and the frequency of truck load applications increase. As the requests for special permits to use bridges by heavy trucks increases, there is a concern over the potential for rapid damage to bridges if the number of such permits is issued frequently and without any control. Furthermore, some bridges have been built for low truck weights (some for as little as 240-kN (54-kip), especially along feeder ramps) than current design load, which is $356-\mathrm{kN}$ (80-kip) in the United States. Hence, some of these bridges may have a shorter life than expected and thus may become subject to premature failure, if the loading spectra contains many occurrences of overloads.

Mohammadi et al. report on a study of fatigue damage in steel girders in bridges with welded cover plates to determine their potential fatigue damage from overloads [5]. The study indicated that the rate of damage accumulation because of overloads can be several magnitudes larger than those because of regular loads. In the long run, the situation may become critical, if the damage from overloads is not controlled. These bridges may then be subject to premature costly repair and replacement.

In this paper, (1) the significance of overloads in causing fatigue damage to bridges is discussed; (2) truck load data have been compiled; and (3) the contribution of overloads to fatigue damage for steel girder bridges made up of welded cover plates is determined using the compiled truck load data. The paper discusses using the expected fatigue life expended (FLE) as an index to determine the impact of overloads on bridge fatigue damage. The FLE is then determined to be an important factor that can be used to modify bridge rating equations as a way of controlling the number of overload permits for a given bridge. It is demonstrated that by limiting the FLE and modifying the bridge rating equations, a reduction in the number of permits is achieved, which in turn will prevent bridges from rapidly approaching the end of their service life. Illustrative examples are provided in the paper to demonstrate the applicability of the modified rating equations along with discussions and conclusions.

\section{Distributions of stress ranges at fatigue critical locations because of overloads}

In this study, the stress range is determined by conducting a series of structural analyses using various truck load data sets obtained from the WIM system. The structural analysis was conducted considering designs conforming to the AASHTO Specifications in terms of load distribution factors, the number of lanes, design requirements, etc [6].

As mentioned earlier, a major portion of the overloaded trucks in the entire truck load population is made up of the vehicle class 9 in the 5-axle truck category. The stress range is computed from the gross vehicle weight (GVW) with the critical axle load configuration shown in Figure 2. The corresponding frequencies for stress ranges are considered and counted based on the fact that usually the passage of a truck will result in one major stress cycle at a given critical location. Figure 3 presents the stress range history at a fatigue-critical location in four bridges in which the span lengths are 21.3 meters (for Bridge \#1), 18.3 meters (for \#2), 15.2 meters (for \#3) and 12.5 meters (for \#4), respectively. The coefficient of variation in stress range is computed to be between $4 \%$ to $12 \%$, indicating the variability in the GVW data. Figure 4 presents the stress range data for Bridge $\# 1$; and as seen, the stress range distribution also has two distinct peaks, as expected.

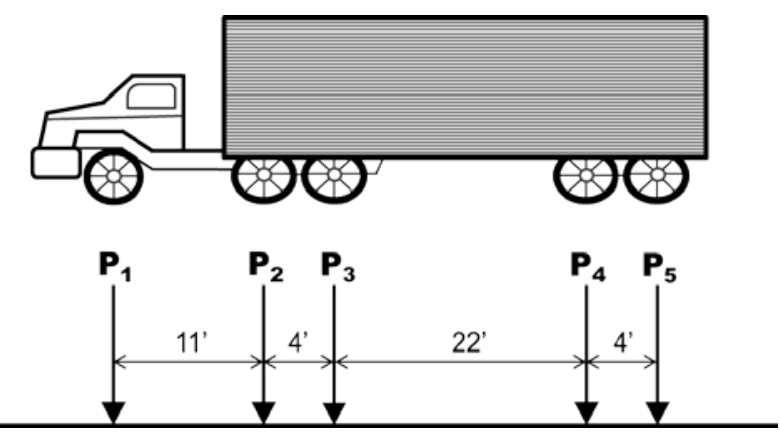

Fig. 2. Type 3-S2 truck configuration (in the United States)

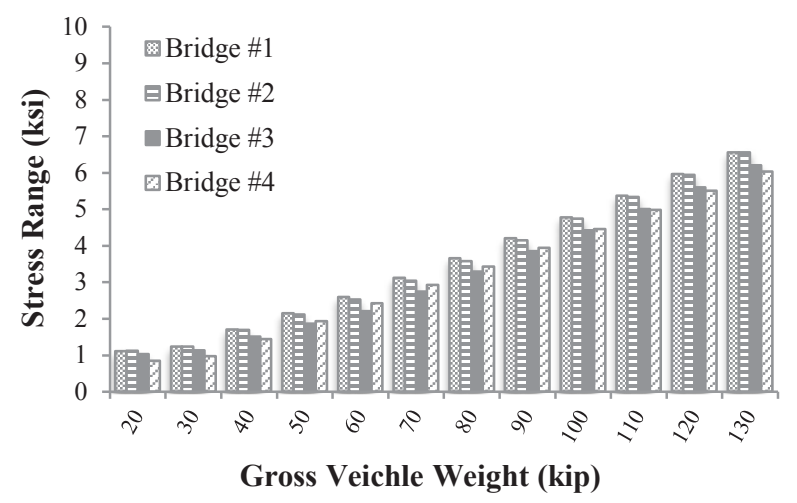

Fig. 3. Stress range history at the critical point on the given bridges 


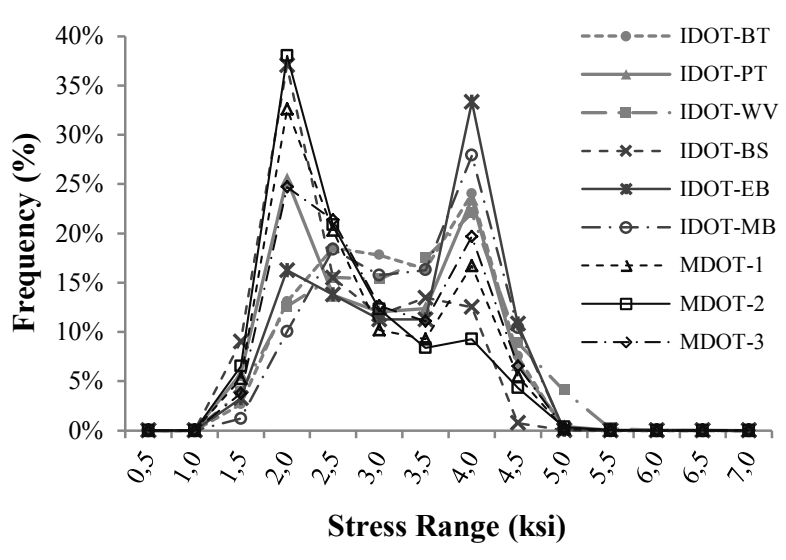

Fig. 4. Stress range distribution for all WIM stations for Bridge $\# 1$

\section{Fatigue damage expected from overloads}

In this paper, the focus is on damage that can occur to bridges in cases where overloads become a frequent occurrence. Fatigue damage from overloads is estimated using the Miner linear-damage rule at a critical location and a detail with a known S-N relationship. Bridges investigated are considered functionally failed when damage at the critical detail equals unity. Using this notion, the bridge fatigue life is then estimated.

To estimate the fatigue damage and the remaining fatigue life, a deterministic or probabilistic method may be used [7]. The probabilistic method allows incorporating uncertainties inherent in Miner damage rule, S-N relation curve, and stress range data in the fatigue damage estimation. Because of its formulation process, the probabilistic process provides fatigue life estimates that are based on specific values of accepted failure probabilities (for example, $2.3 \%$ for nonredundant structures). The deterministic method, on the other hand, offers life estimations in simple forms that can readily be used for practical applications without specific reference to any probability of failure. In this paper, the deterministic method is used.

The damage equation is as follows:

$$
D=\sum n_{i} / N_{i} \quad(i=1 \text { to } k)
$$

In which $D$ is the total damage accumulated, $k$ is the number of stress ranges developed from the load population on a given bridge, $n_{i}$ is the number of applied cycles for $i^{\text {th }}$ stress range, and $N_{i}$ is the total number of cycles to failure of the $i^{\text {th }}$ stress range. The number of applied cycles is from the truck load population as indicated earlier; and the total number of cycles to failure is defined from S-N curve that is componentdependent. The following equation is used to represent the S-N curve:

$$
N=C / S^{m}
$$

In which $N$ is the number of cycles to failure corresponding to stress range $S$, and $C$ and $m$ are constants specific to the structural detail (see for example, Munse et al [8]). For steel beams with welded cover plates corresponding to the fatigue detail categories $\mathrm{E}$ and $\mathrm{E}^{\prime}$ in AASHTO manual, $C$ and $m$ are 4.218E09 and 3.256, respectively [9]. This type of detail is used in this paper.

\section{Determination of fatigue damage potentials from overloads, FLE}

The computation of the stress range at a fatigue-critical location requires a structural analysis of the bridge when it is subjected to truck axle loads. It is noted that the Miner damage rule assumes constant load amplitude and a linear formulation for the computation of fatigue damage. Since the stress data has many ranges each with its own number of cycles, using the damage equation incrementally, damage for each stress range is computed individually for the specific number of cycles for that range. The total damage is the summation of these incremental damage values.

The fatigue damage is estimated for a one-year period and then extrapolated considering traffic growth in future years. The accumulation of damage over 25, 50, 75 , and 100 years can be obtained using the annual damage with and without traffic growth over this period. In this paper, specifically, the stress ranges of Bridge \#1 are used as a demonstration of the procedure in computing the typical trend in fatigue life expended (FLE). Tables 1 and 2 summarize the FLE results for Bridge \#1 with and without traffic growth. Figure 5 represents the annual fatigue damage due to overloads as a proportion of total fatigue damage when the entire load population is considered.

As seen, the fatigue life expended (FLE) varies depending on the volume of traffic and the percentage of overloads in the entire truck load population. Considering the data from all WIM stations, it is observed that overloads will use up about $0.2 \%$ to $7.8 \%$ and $0.6 \%$ to $23 \%$ of the fatigue life of the bridge component in 25 and 75-year periods, respectively. These are if no annual traffic increase is considered. According to a NCHRP 2016 report, from 2005 to 2012, a trend in oversize and overweight transportation showed approximately $3 \%$ annual increase in the truck traffic [10]. Thus, it is imperative to consider some level of steady traffic growth over a long period of time. For example, considering a $2 \%$ annual increase in truck traffic, the damage over a 25 -year period for Bridge \#1 can amount to about $0.26 \%$ to $10 \%$ of the fatigue life of the component. In addition, for 50 and 75 -year periods, the cumulated fatigue damage potentials significantly increase with a $2 \%$ annual traffic growth. The percentage reduction in fatigue life may be especially important for older bridges that are designed for a lower truck load than current design requirements. For these bridges, any additional reduction in fatigue life may be critical resulting an early replacement or costly repairs. 
Table 1. Cumulative fatigue damage over years without a traffic growth- Bridge \#1.

\begin{tabular}{|c|c|c|c|c|c|}
\hline \multirow{2}{*}{$\begin{array}{l}\text { Location } \\
\text { of Station }\end{array}$} & \multirow{2}{*}{$F L E_{1 \mathrm{yr}}^{\mathrm{a}}$} & \multicolumn{4}{|c|}{ Without Traffic Growth } \\
\hline & & $F L E_{25 \mathrm{yrs}}$ & $F L E_{50 \mathrm{yrs}}$ & $F L E_{75 \mathrm{yrs}}$ & $F L E_{100 \mathrm{yrs}}$ \\
\hline IDOT-BT & 0.00099 & 0.0248 & 0.0496 & 0.0743 & 0.0991 \\
\hline IDOT-PT & 0.00094 & 0.0235 & 0.0471 & 0.0706 & 0.0941 \\
\hline IDOT-WV & 0.00312 & 0.0781 & 0.1562 & 0.2342 & 0.3123 \\
\hline IDOT-BS & 0.00008 & 0.0020 & 0.0040 & 0.0060 & 0.0080 \\
\hline IDOT-EB & 0.00076 & 0.0191 & 0.0381 & 0.0572 & 0.0762 \\
\hline IDOT-MB & 0.00189 & 0.0472 & 0.0945 & 0.1417 & 0.1889 \\
\hline MDOT-1 & 0.00016 & 0.0040 & 0.0079 & 0.0119 & 0.0159 \\
\hline MDOT-2 & 0.00074 & 0.0185 & 0.0370 & 0.0554 & 0.0739 \\
\hline MDOT-3 & 0.00107 & 0.0269 & 0.0537 & 0.0806 & 0.1075 \\
\hline
\end{tabular}

Table 2. Cumulative fatigue damage over years with a traffic growth $(2 \%)$ - Bridge \#1.

\begin{tabular}{lccccc}
\hline \hline \multirow{2}{*}{$\begin{array}{l}\text { Location } \\
\text { of Station }\end{array}$} & $F L E_{1 \mathrm{yr}}^{\mathrm{a}}$ & \multicolumn{5}{c}{ With Traffic Growth (2\%) } \\
\cline { 3 - 6 } & & $F L E_{25 \mathrm{yrs}}$ & $F L E_{50 \mathrm{yrs}}$ & $F L E_{75 \mathrm{yrs}}$ & $F L E_{100 \mathrm{yrs}}$ \\
\hline IDOT-BT & 0.00099 & 0.0317 & 0.0838 & 0.1693 & 0.3094 \\
IDOT-PT & 0.00094 & 0.0301 & 0.0796 & 0.1607 & 0.2939 \\
IDOT-WV & 0.00312 & 0.1000 & 0.2641 & 0.5334 & 0.9751 \\
IDOT-BS & 0.00008 & 0.0026 & 0.0068 & 0.0137 & 0.0250 \\
IDOT-EB & 0.00076 & 0.0244 & 0.0645 & 0.1302 & 0.2380 \\
IDOT-MB & 0.00189 & 0.0605 & 0.1598 & 0.3227 & 0.5899 \\
MDOT-1 & 0.00016 & 0.0051 & 0.0135 & 0.0272 & 0.0496 \\
MDOT-2 & 0.00074 & 0.0237 & 0.0625 & 0.1263 & 0.2308 \\
MDOT-3 & 0.00107 & 0.0344 & 0.0909 & 0.1835 & 0.3355 \\
a FLE is the fatigue life expended. & & & \\
\hline
\end{tabular}

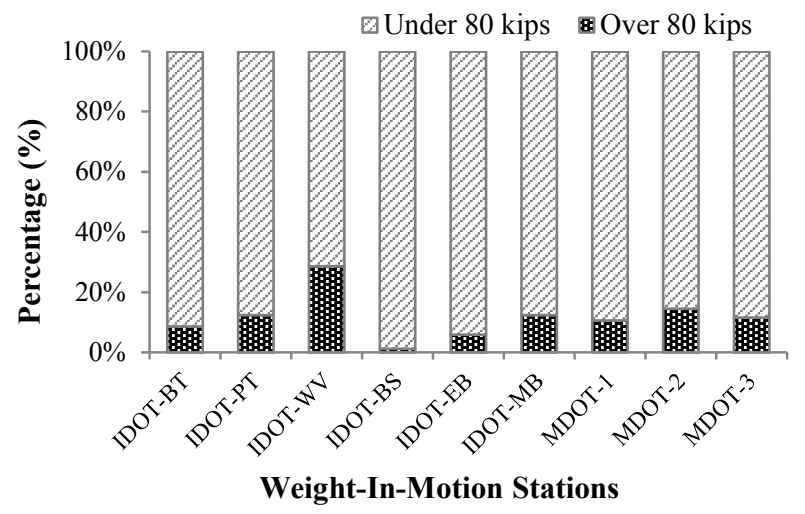

Fig. 5. Proportions of the annual fatigue damage due to overloads for Bridge \#1

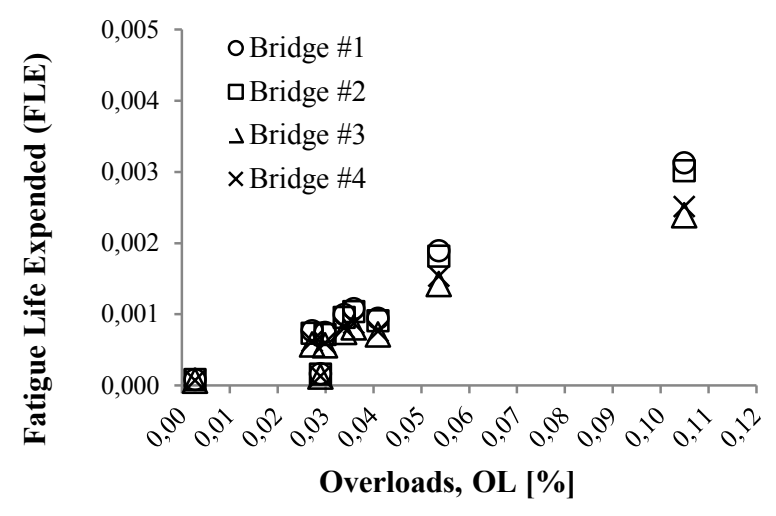

Fig. 6. Annual fatigue damage from overloads in terms of the percentage of overload in the entire truck load population

Figure 6 shows the significance of percentage of overload trucks in the truck population. It shows that the relationship between the fatigue life expended (FLE) and the percentage of overloads (in the entire truck load population) is almost linear (at least for the percentages considered in this study, i.e., up to $12 \%$ ). This type of linear relationship may be helpful in offering a straightforward approach in incorporating the FLE as an index in bridge rating equations to account for the significance of fatigue damage from overloads.

\section{Illustrative Examples and Discussions}

The fatigue life expended (FLE) is determined to be an important factor that can be used to modify bridge rating equations to account for the significance of fatigue damage from overloads. It is demonstrated that by limiting the FLE and modifying the bridge rating equations, a reduction in the number of permits is achieved, which in turn will prevent bridges from rapidly approaching the end of their service life. It is noted that the rating factor represents the safety and serviceability of the bridge by evaluating the effect of passage of the overload truck for the purpose of the permit-load rating. In general, a rating of greater than 1 is considered satisfactory unless otherwise specified. A modification factor, $\alpha_{F L E}$, is proposed in the bridge rating equation to account for the significance of the fatigue damage potentials for the overloads [4]. This modification factor is determined based on the cumulative fatigue damage and is formulated as a bilinear function in terms of a current age of bridge $(a)$ and the percentage of overloads in the truck load population $(O L)$. The modification factor is used in the Load and Resistance Factor Rating (LRFR) method as given in the AASHTO's Manual for Bridge Evaluation [9].

\subsection{Examples}

For illustrative purposes, a simply supported steel girder bridge with a welded cover plate (Category $\mathrm{E}$ in fatigue details) is considered. The span length of the bridge is $21.3-\mathrm{m}(70-\mathrm{ft})$. The parameters used in this example are: $\mathrm{ADTT}=2623$ trucks/day and $O L=0.105(10.5 \%), \varphi$ 
(capacity reduction factor) $=0.95, \gamma_{D C}=1.25, \gamma_{D W}=1.5$, $\gamma_{L}=1.441$ (these are the load factors), IM $=1.2$ (for minor surface deviations), traffic growth $=2 \%$, fatigue strength coefficient $=11 \mathrm{E} 08$ (for fatigue detail category E). These parameters were used for estimating (1) the remaining fatigue life of the example bridge using the equation provided by the Manual for Bridge Evaluation [9]; and (2) the rating with and without the modification factor. Several different ages for the bridge are considered (25, 50 and 65 years) to estimate and compare the remaining fatigue life and the rating factor. The remaining fatigue life is presented for the 25 and 50year bridges; while the modified rating values are shown for the 50 -year bridge as well as for a case where the age is as long as 65 years.

The example bridge was analyzed using the 5-axle truck configuration as mentioned earlier. Structural analyses were conducted to obtain the bending moment and the corresponding stress ranges at the critical component in the steel girders. Figures 7 and 8 present the results for the remaining fatigue life and the rating factor, respectively for the two different bridge ages.

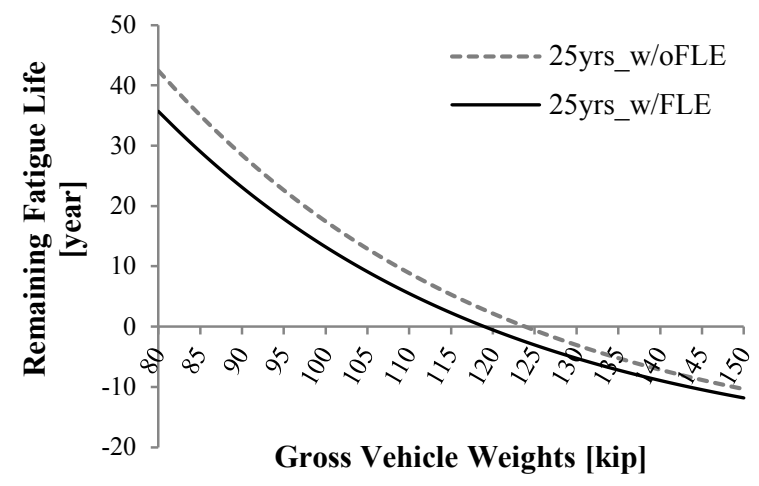

(a) Bridge with 25 years of life

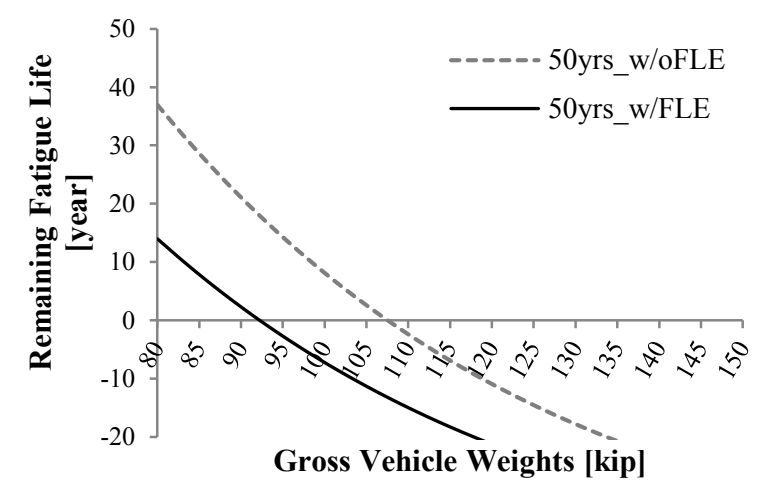

(b) Bridge with 50 years of life

Fig. 7. Remaining fatigue life with and without FLE for two different ages for a given bridge

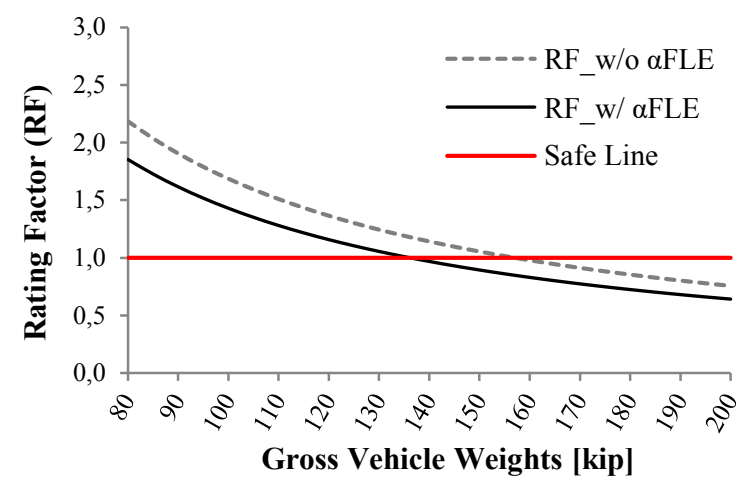

(a) Bridge with 50 years of life

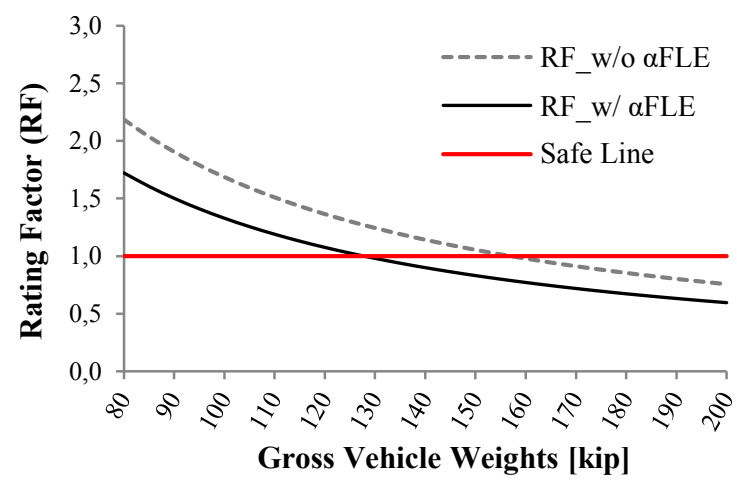

(b) Bridge with 65 years of life

Fig. 8. Rating factor with and without the modification factor, $\alpha_{F L E}$, for two different ages for a given bridge

\subsection{Discussions}

As expected, the remaining fatigue life for the 25-year and 50-year bridges decreases with the growth vehicle weight as shown in Figure 7. It is noted that a negative fatigue life is only a mathematical estimate and corresponds to a practical case when the bridge age exceeds the predicted cyclic life. This situation happens when the gross vehicle weight is greater than $556-\mathrm{kN}$ (125-kip) for the 25-year bridge and 480-kN (108-kip) for the 50-year bridge without consideration for the fatigue life expended (FLE). However, if FLE with traffic growth for overloads is considered, the remaining fatigue life decreases even more dramatically compared with the cases where FLE is not considered. The 50-year bridge has a significantly more reduction in its service life. This indicates that when the bridge ages, the remaining fatigue life dramatically decreases. This is mainly attributed to the traffic growth and to a lesser extent to the percentage of the overloads in the truck load population. As shown in Figure 7, if the overloaded truck with only 414-kN (93-kip) applies on the 50-year bridge, the negative remaining fatigue life is resulted and field inspection has to be initiated to ascertain the condition of the critical details for any significant cracks.

The values of the modification factor, $\alpha_{F L E}$, were determined to be 0.1790 for the 50-year bridge and 0.2687 for the 65 -year bridge. In this example bridge, the rating values are satisfactory without the modification factor if the gross vehicle weight is less than $689-\mathrm{kN}$ 
(155-kip) for both cases. However, the ratings with considerations for the fatigue damage result in values less than 1 for overloads at 601-kN (135-kip) and 556$\mathrm{kN}$ (125-kip), for the 50-year and 65-year bridges, respectively as shown in Figure 8. As is evident from Figure 8, using the modification factor has resulted in certain number of overloads to be disallowed due to the fatigue damage potentials. This in turn indirectly imposes a limit on the number of permits.

\section{Conclusions}

The following presents the main conclusions from this study.

1) Fatigue damage potentials from overloads may become significant, if the number of special permits for overloads is issued frequently and without any control.

2) Fatigue life expended (FLE) varies depending on the volume of traffic (ADTT) and the percentage of overloads in the entire truck load population $(O L)$. FLE shows a linear trend with the percentage of overloads $(O L)$.

3) This linear relationship offers a straightforward equation for incorporating FLE in the bridge condition assessment.

4) Incorporating the FLE in the load rating equations, may be an indirect method in limiting the number of permits for overloads.

The assistance provided by David C. Thompson of the Illinois and Thomas Foltz of the Michigan Department of Transportation, respectively, in making the WIM data available to the authors is appreciated.

\section{References}

1. J. Mohammadi, N. Shah, J. Trans. Eng. ASCE, Statistical evaluation of truck overloads, 118 (5), 651-665 (1992)

2. R.E. Snyder, G.E. Likins, F. Moses, Loading spectrum experienced by bridge structures in the United States, Report RD-851012, (FHWA, Washington DC. 1985)

3. D.H. Timm, S.M. Tisdale, R.E. Turochy, J. Trans. Eng. ASCE, Axle load spectra characterization by mixed distribution modeling, 132 (2), 83-88 (2005)

4. B. Jang, J. Mohammadi, Brdg. Strc., Bridge rating modification to incorporate fatigue damage from truck overloads, 13 (2-3), 101-107 (2017)

5. J. Mohammadi, R. Polepeddi, J. Brdg. Eng. ASCE, Bridge rating with consideration for fatigue damage from overloads, 5 (3), 259-265 (2000)

6. AASHTO, AASHTO LRFD Bridge Design Specifications, $8^{\text {th }}$ edition, (AASHTO, Washington DC. 2017)

7. J. Mohammadi, S.A. Guralnick, R. Polepeddi, FFRC of SEI of ASCE, Use of Stress Range Data in Fatigue Reliability Assessment of Highway Bridges, in NDT Methods Applied to Fatigue Reliability Assessment of Structures (J. Mohammadi, ed), 56-71 (2004)

8. W. Munse, T. Wilbur, M. Tefralian, K. Nicoll, K. Wilson, Fatigue characterization of fabricated ship details for design, Report SSC-318, (Ship Strc. Cmt. Washington D.C. 1983)

9. AASHTO, Manual for bridge evaluation, $2^{\text {nd }}$ edition, (AASHTO, Washington DC. 2011)

10. CPCS, Perkins Motor Transport, Inc., Portscape, Inc., Multi-State, Multimodal, Oversize/Overweight Transportation, NCHRP Report 830, (TRB, Washington DC. 2016) 